\title{
A copy number variation in human NCF1 and its pseudogenes
}

Tiffany Brunson, Qingwei Wang, Isfahan Chambers, Qing Song*

\begin{abstract}
Background: Neutrophil cytosolic factor-1 (NCF1) is a component of NADPH oxidase. The NCF1 gene colocalizes with two pseudogenes (NCF1B and NCF1C). These two pseudogenes have a GT deletion in exon 2, resulting in a frameshift and an early stop codon. Here, we report a copy number variation (CNV) of the NCF1 pseudogenes and their alternative spliced expressions.

Results: We examined three normal populations (86 individuals). We observed the 2:2:2 pattern (NCF1B:NCF1: NCF1C) in only 26 individuals. On average, each African- American has $1.4 \pm 0.8$ (Mean \pm SD) copies of NCF1B and $2.3 \pm 0.6$ copies of NCF1C; each Caucasian has $1.8 \pm 0.7$ copies of NCF1B and $1.9 \pm 0.4$ copies of NCF1C; and each Mexican has $1.6 \pm 0.6$ copies of NCF1B and $1.0 \pm 0.4$ copies of NCF1C. Mexicans have significantly less NCF1C copies than African-Americans ( $p=6 \mathrm{e}-15)$ and Caucasians $(p=3 \mathrm{e}-11)$. Mendelian transmission of this CNV was observed in two CEPH pedigrees. Moreover, we cloned two alternative spliced transcripts generated from these two pseudogenes that adopt alternative exon-2 instead of their defective exon 2. The NCF1 pseudogene expression responded robustly to PMA induction during macrophage differentiation. NCF1B decreased from 32.9\% to $8.3 \%$ in the CDNA pool transcribed from 3 gene copies. NCF1Ys also displayed distinct expression patterns in different human tissues.
\end{abstract}

Conclusions: Our results suggest that these two pseudogenes may adopt an alternative exon-2 in different tissues and in response to external stimuli. The GT deletion is insufficient to define them as functionless pseudogenes; this CNV may have biological relevance.

\section{Background}

Recent genomic studies suggested that gene duplication occurred frequently and in variable numbers during the recent history of human populations, which has led to de novo formations of copy number variation (CNV) [1]. Presumably due to positive selection, genes encoding certain protein categories are particularly enriched in CNVs, such as those involved in processes related to environmental responses [1-8]. In this process, duplicated genes are thought to be the "successful" copies; pseudogenes are those "unsuccessful" duplicates retained in the genome [1].

Neutrophil cytosolic factor 1 (NCF1, also called $\mathrm{p} 47^{\text {phox }}$, for phagocyte oxidase), is a crucial component of NADPH oxidase [9]. This enzyme catalyzes the production of microbicidal superoxide in phagocytes such

\footnotetext{
* Correspondence: qsong@msm.edu

Cardiovascular Research Institute, Morehouse School of Medicine, Atlanta, Georgia, USA
}

as neutrophil and plays a vital role in host defense against microbial pathogens [10,11]. A 2-bp GT deletion in exon 2 of the NCF1 gene causes chronic granulomatous disease (CGD) in humans [12,13]. NCF1 is expressed in many cell types and may play a role in many other diseases [14-19].

The human NCF1 gene is located at 7q11.23, the Williams Beurens Syndrome region [20-22], accompanied by two nearly identical (>99.5\%) pseudogenes (NCF1B and NCF1C) which presumably arose by gene duplication [23]. These two pseudogenes have the same signature sequence as the one in the NCF1 gene responsible for CGD, the 2-bp GT deletion in exon $2[13,23,24]$. This mutation leads to a frameshift and a premature stop codon and thus these two gene duplicates were categorized as pseudogenes. It has been noticed that the NCF1 $\Psi /$ NCF1 ratio vary in human individuals, and it was believed some NCF1 $\Psi$ gene copies contain the wild-type NCF1 exon 2 sequence [22,25]. 
In this study, we examined the copy numbers of NCF1 pseudogenes in human populations and found a copy number variation $(\mathrm{CNV})$. Our additional data revealed that these two pseudogenes can generate RNA transcripts that skip over their defective exon 2 by alternative splicing.

\section{Results}

\section{Copy Number Variation of NCF1 and NCF1 $\Psi$}

There are three large highly homologous duplicons at the NCF1 locus (Figure 1). According to their chromosomal positions (UCSC Human Assembly 2006 March, chr7:72, 272,547-72,287,915 [pseudogene], chr7:73,826,245-73, 841,595 [NCF1], chr7:74,210,381-74,225,753 [pseudogene]), we designated two NCF1 pseudogene duplicons as NCF1B and NCF1C, respectively. These duplicons share a $106-\mathrm{kb}$ sequence with $>99.5 \%$ similarities spanning from $-45 \mathrm{~kb}$ at 5 -end to $+46 \mathrm{~kb}$ at 3 '-end regarding the NCF1 coding region. NCF1 or NCF1C duplicons share an additional 3'-flanking sequence until $+82 \mathrm{~kb}$ (Figure 1). In contrast to the human genome, NCF1 is a single-copy gene in the reference genomes of all other species, such as chimpanzee, rhesus monkey, rat and mouse (UCSC Genome Assemblies). The phylogenetic tree revealed that NCF1B and NCF1C duplicated after they arose from the NCF1 gene (Figure 2).

To determine the relative copy numbers of NCF1B, NCF1, and NCF1C, we genotyped the genomic DNA of human subjects at two particular positions, the signature 2-bp GT deletion in exon 2, and an A/G substitution in exon-9, in which NCF1B and NCF1 has an A allele, and NCF1C has a G allele (Figure 1 and Additional file 1). Pyrosequencing is a high-throughput technology that can be used for accurate determination of the allele frequency in pooled DNA [26]. Based on the pyrogram peak heights, we assessed the allele composition of each individual with the PyroMarkID Software v1.0 (Additional file 2).
We analyzed 86 non-related individuals (32 AfricanAmericans [AA], 30 Caucasians [Cau] and 24 Mexicans [Mex]). Totally we observed 6 different NCF1 $/$ NCF1 ratios in these normal populations (Figure $3 \mathrm{a}$ and Additional file 3). The 4:2 ratio (4 pseudogenes \& 2 NCF1 in each genome) are predominant in AA (71.9\%) and Caucasians (56.1\%), but rare in Mexicans (4.2\%), instead, the $3: 2(50.0 \%)$ and $2: 2(41.7 \%)$ genotypes are common in Mexicans. Interestingly, the Mexican population has an additional genotype (4.2\%), 1:2, which contains only one pseudogene copy in each genome.

Using the genotyping pyrograms at exon 2 (GT/GTGT/ GT) $[\mathrm{NCF} 1 \mathrm{~B} / \mathrm{NCF} 1 / \mathrm{NCF} 1 \mathrm{C}]$ and exon 9 (A/A/G), we were able to further dissect the copy numbers of two pseudogenes in each genome (see Additional file 3). On average, each AA individual has $1.4 \pm 0.8$ (Mean \pm SD) copies of NCF1B, $2.1 \pm 0.7$ copies of NCF1, and $2.3 \pm$ 0.6 copies of NCF1C; each Caucasian has $1.8 \pm 0.7$ copies of NCF1B, $2.1 \pm 0.3$ copies of NCF1, and $1.9 \pm$ 0.4 copies of NCF1C; and each Mexican genome has $1.6 \pm 0.6$ copies of NCF1B, $2.1 \pm 0.3$ copies of NCF1, and $1.0 \pm 0.4$ copies of NCF1C. There is a significant difference on the copy number of NCF1C among these 3 human populations (Figure 3c and 3d), in which Mexicans have significantly less copies of NCF1C than AA $(p=6 \mathrm{e}-15)$ and Cau $(p=3 \mathrm{e}-11)$. There is also a significant difference on the copy number of NCF1B between $\mathrm{Cau}$ and AA $(p=0.033)$.

Epstein-Barr virus (EBV) transformed lymphoblastoid cells lines are widely used as a genomic resource for many human genetic studies. However, chromosomal instability, which can cause a duplication or deletion in the host or viral genome sequences flanking the integration sites, is increased by viral integration [27]. In order to eliminate the possibility that this CNV is just an artifact in lymphoblastoid cell lines, we analyzed 48 genomic DNA samples directly extracted from human

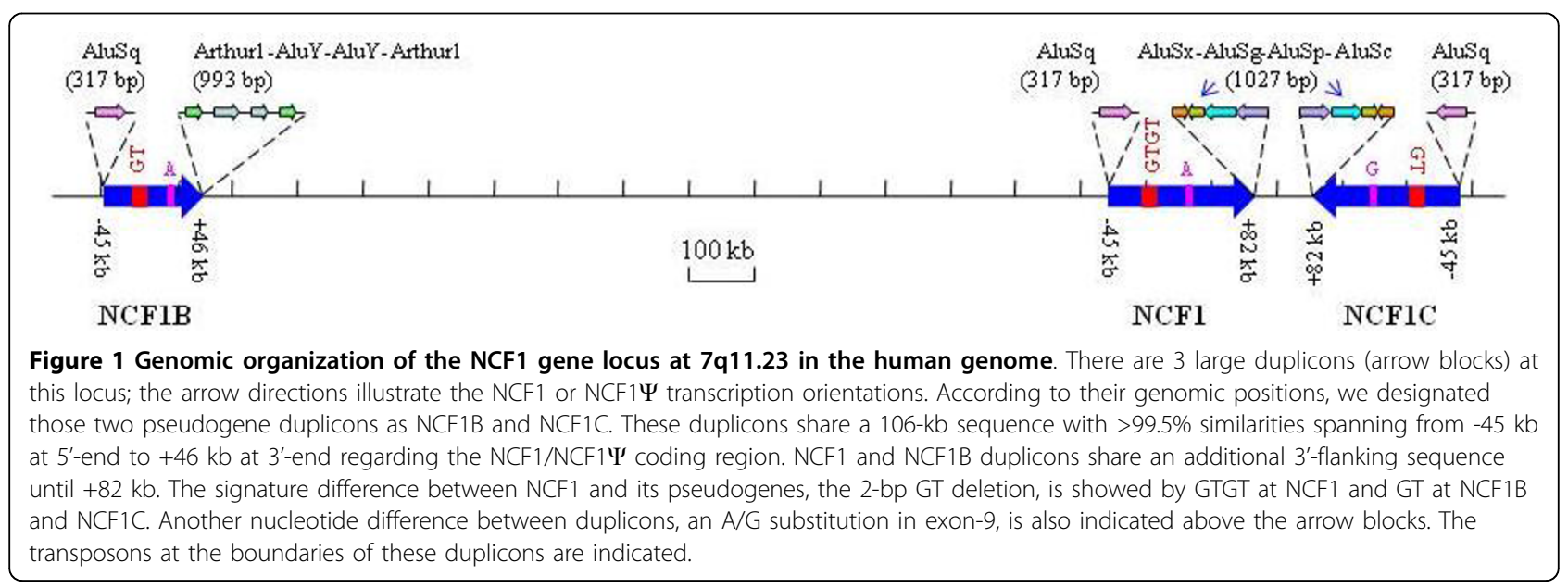




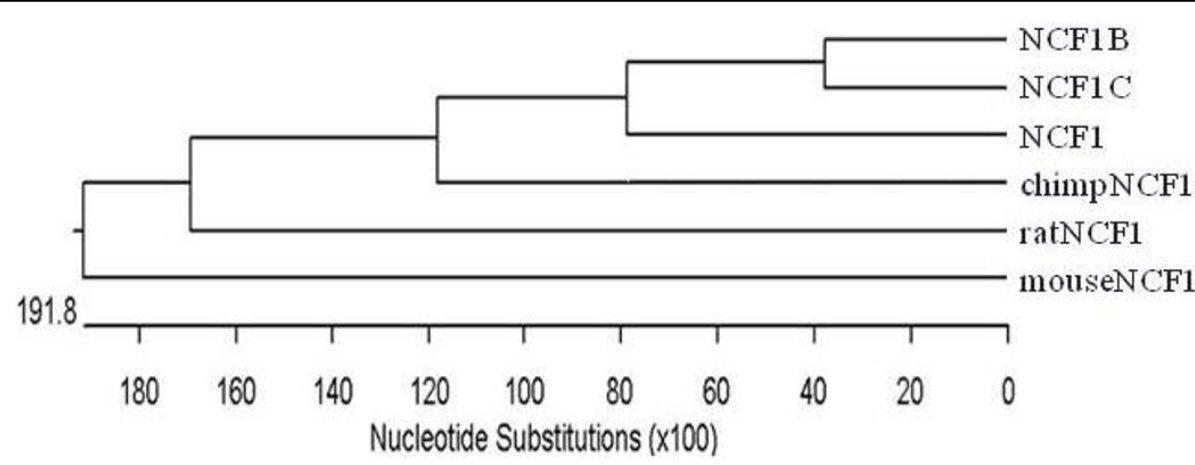

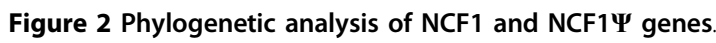

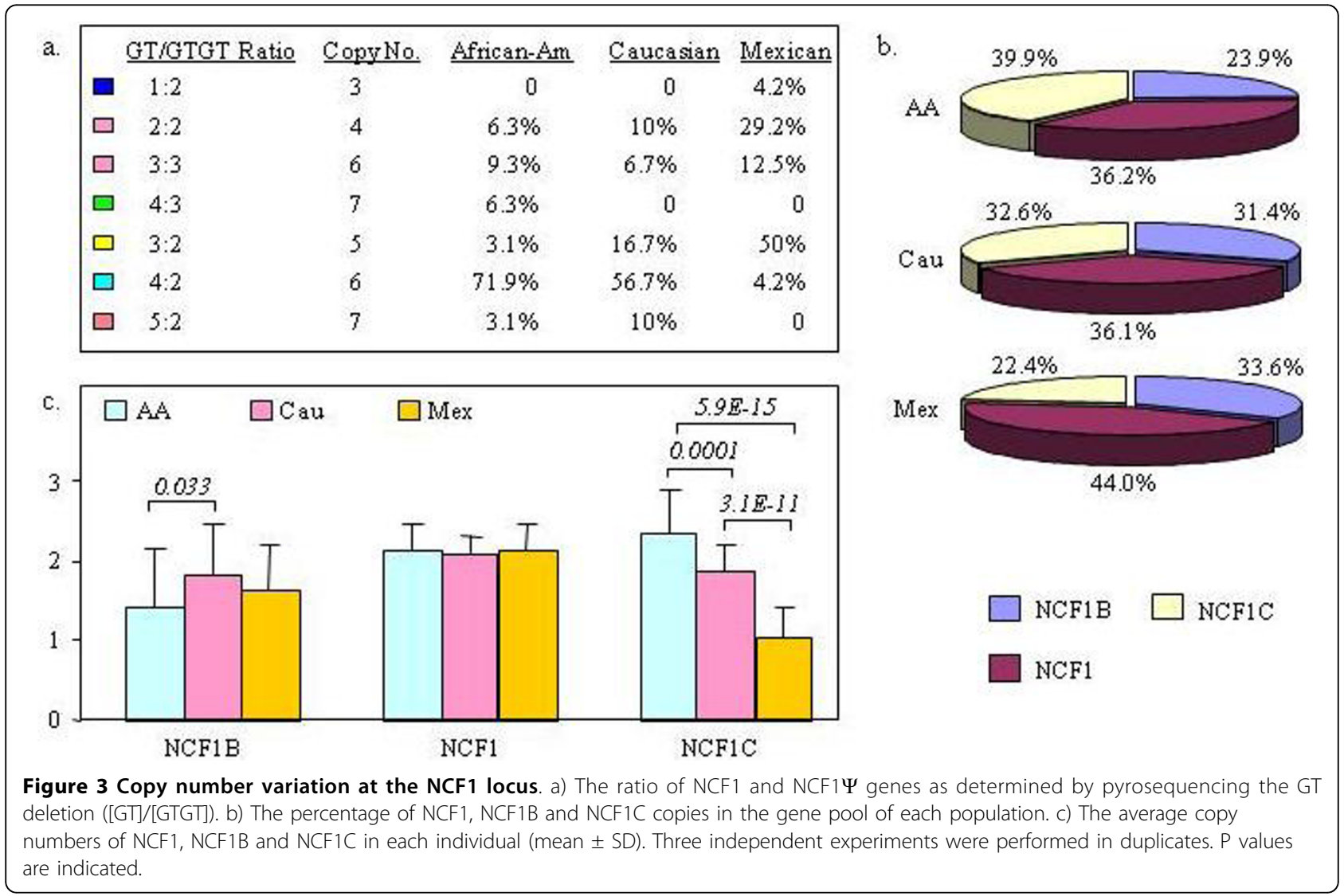

peripheral white blood cells (Additional file 4). Collectively 4 individuals had 5:2 ratios $(2.41+0.058), 18$ individuals had 2:1 ratios $(1.97+0.141), 23$ individuals had $3: 2$ ratios $(1.53+0.141)$ and 3 individuals had 1:1 ratios. This data confirmed the presence of this CNV.

\section{Heritability of NCF1 Copy Number Variation}

Two large CEPH families were used to examine the heritability of this CNV detected at the NCF1 locus (Figure 4). The majority of the members in family 1331 have a $2: 1 \Delta$ GT/GTGT ratio as represented by a $4: 2$ ratio in a diploid genome, which was further determined as a 1:2:3 proportion (NCF1B: NCF1: NCF1C); one family member, the paternal grandmother, has a $0: 2: 3$ ratio. Family 1362 is particularly interesting as both maternal and paternal lineages have very different NCF1B:NCF1:NCF1C ratios. The paternal lineage has an unvarying $4: 2$ ratio $(1: 2: 3)$ where as the maternal lineage has a continuous 2:2 (0:2:2) ratio. Based on the potential haplogenotypes deduced from the pedigrees, we 


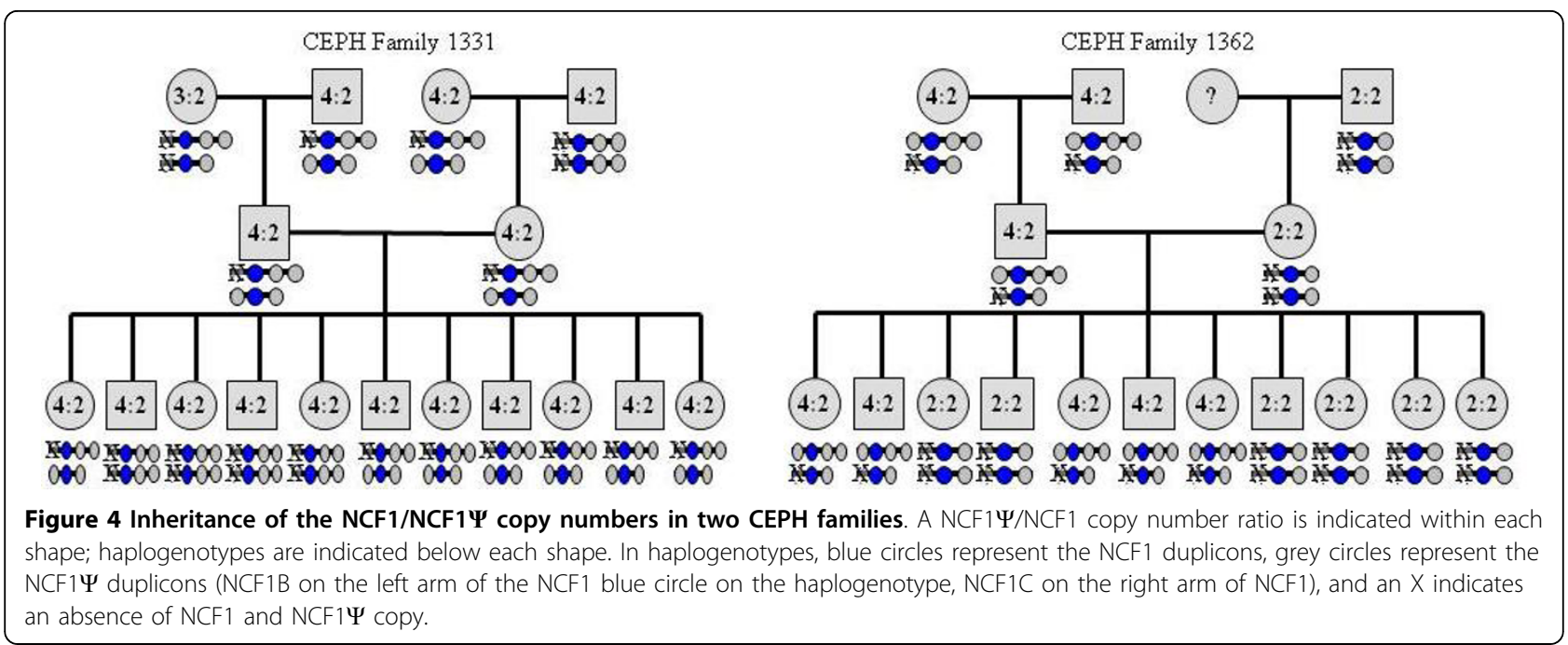

observed a clear allele transmission pattern of this $\mathrm{CNV}$ in both families, suggesting a heritability of this CNV; however, our data cannot exclude the de novo formation of new copy numbers of this $\mathrm{CNV}$ in other families. The clear inheritance of the copy numbers in these two large pedigrees also suggests that there is no large experimental bias on the copy number measurement.

\section{Transcription and Alternative Splicing of NCF1 $\Psi$ and NCF1 Genes}

We explored if these two NCF1 pseudogenes are transcriptionally active. Pyrosequencing was used to quantify the NCF1/NCF1 $\Psi$ s compositions in the mRNAs of 14 single-donor lymphoblastoid cell lines by genotyping the signature 2-bp GT deletion in cDNA (Figure 5). Interestingly, although NCF1 $\Psi$ s have more copies than NCF1 in each individual, they made much less GT-containing transcripts than NCF1. For example, in the individual-6 who has 4 copies of NCF1 1 s and 2 copies of NCF1, pseudogenes collectively only contributed the amount of transcripts equal to half of the amount from the NCF1 gene copy (as revealed by the ratio 0.5:1 in cDNA).

By PCR, cloning and direct DNA sequencing, we have experimentally discovered two novel alternative exons (GenBank: GU215077, GU215078) located in the intron1 (Additional file 5). Neither of these two new alternative splicing transcripts used the GT-containing exon-2 (Figure 6b), and both of them were made from the NCF1Ys copies as revealed by their nucleotide sequences (Figure 6c). We have searched for putative open reading frames with the NCBI ORF Finder. Sub1 showed a continuous ORF without a stop codon, thus the full-length transcript containing this alternative splicing pattern may produce a long ORF. Sub2 contains

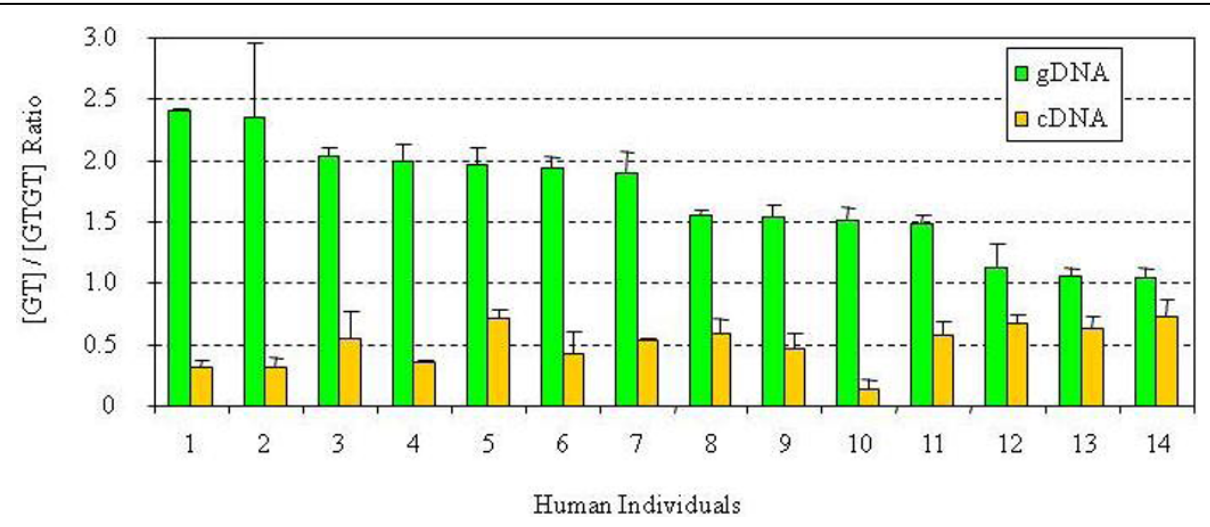

Figure 5 Relative quantities of expression of NCF1 and its pseudogene. The relative proportion of gene expression of NCF1 and NCF1 (GTGT or GT containing transcripts) was measured by genotyping the CDNA samples from LCLs of 14 human individuals at the signature GT deletion. Two pseudogenes collectively contribute to lesser amount of transcript than from one NCF1 gene copy alone (0.3-0.7):1 [NCF1 1 /NCF1, the $[G T] /[G T G T]$ ratio) (yellow bars). The $[G T] /[G T G T]$ ratios in genomic DNA are shown by the green bars. Three independent experiments were performed in duplicates. 


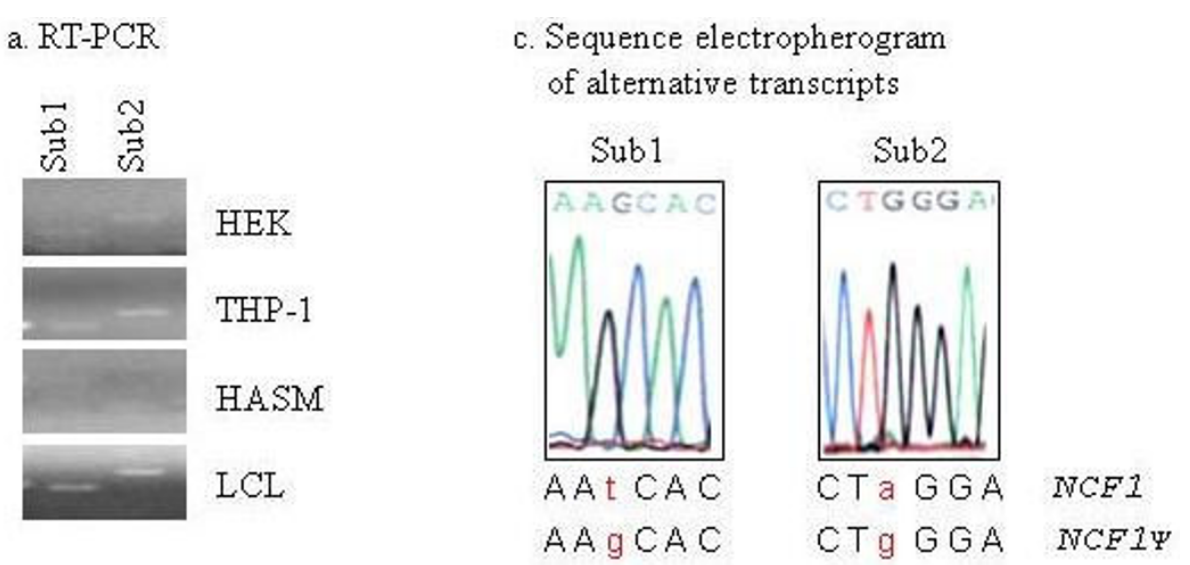

\section{b. Alternative exon-2}

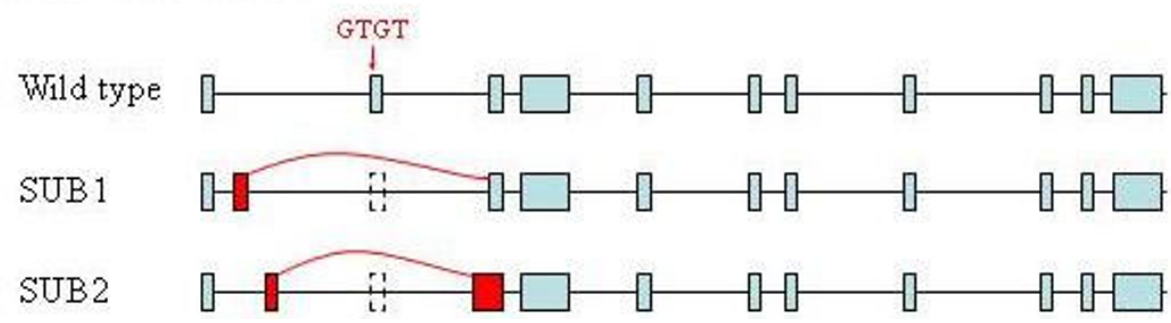

Figure 6 Alternative splicing of the NCF1 pseudogenes. a) RT-PCR detection of two novel pseudogene-specific exons. b) The alternative splicing pattern (sub1 and sub2) using these two new exons, both splicing isoforms skip over the defective exon 2. c) Sequencing electropherogram of sub1 and sub2, the single nucleotide substitutions between NCF1 and its pseudogenes revealed that these two alternative exons were transcribed from the pseudogene copies. HEK, human embryonic kidney cell line; THP-1, a monocyte cell line; HASM, human aortic smooth muscle cells; LCL, lymphoblastoid cell line.

three predicted ORF, but all have a stop codon before its last nucleotide (Additional file 6).

\section{NCF1I and NCF1 Transcription in Monocyte Differentiation}

Monocytes/Macrophages have been implicated in atherosclerosis [28]. After treatment with $800 \mathrm{ng} / \mathrm{ml}$ PMA for $12 \mathrm{hrs}$, the monocytes became adherent and acquired a macrophage-like phenotype. Both monocytes and macrophages have NCF1B:NCF1:NCF1C genomic copy number ratios of 2:2:2 (Figure 7a). After macrophage differentiation, quantitative RT-PCR experiments (RTqPCR) were performed to measure the transcripts with the "GT-containing" exon 2 using primers that recognize all three NCF1 genes. The results showed that the NCF1/NCF1 $\Psi$ s total GT-containing expression is slightly upregulated 1.34 -fold (GAPDH, $\mathrm{p}=0.024$ ) to 1.82 fold $(\beta$-actin, $\mathrm{p}=0.006$ ) (Figure $7 \mathrm{~b}$ ). However, the relative contributions of each pseudogenes and the NCF1 gene copy to the GT-containing transcript pool were altered dramatically. NCF1B decreased from $32.9 \%$ to $8.3 \%$, whereas NCF1 increased from $52.7 \%$ to $80.4 \%$ (Figure 7c).

\section{NCF1Y Expressions in Human Tissues}

We measured the NCF1B:NCF1:NCF1C transcript ratios by pyrosequencing the signature GT deletion in cDNAs generated from different human tissues. We observed that NCF1B and NCF1C expressions (GT-containing transcripts) varied dramatically in different human tissues (Figure 8). For example, skin produced the highest contribution of NCF1B relative to NCF1 expression, whereas pancreas produced the least amount of NCF1B. Spleen and lymph node produced the highest contribution of NCF1B, whereas lung and brain produced the least amount of NCF1C.

\section{Discussion}

In this study, we report the existence of a NCF1 pseudogene CNV in human. The pseudogene copy numbers are apparently different among three human populations (African-Americans, Caucasians, and Mexicans). The CNV existence is validated by observance in genomic DNA extracted directly from human peripheral white blood cells. The NCF1 CNV inheritance found in the two family pedigrees, suggest that the chromosomal instability at this locus may not be high. Our phylogenetic analysis 


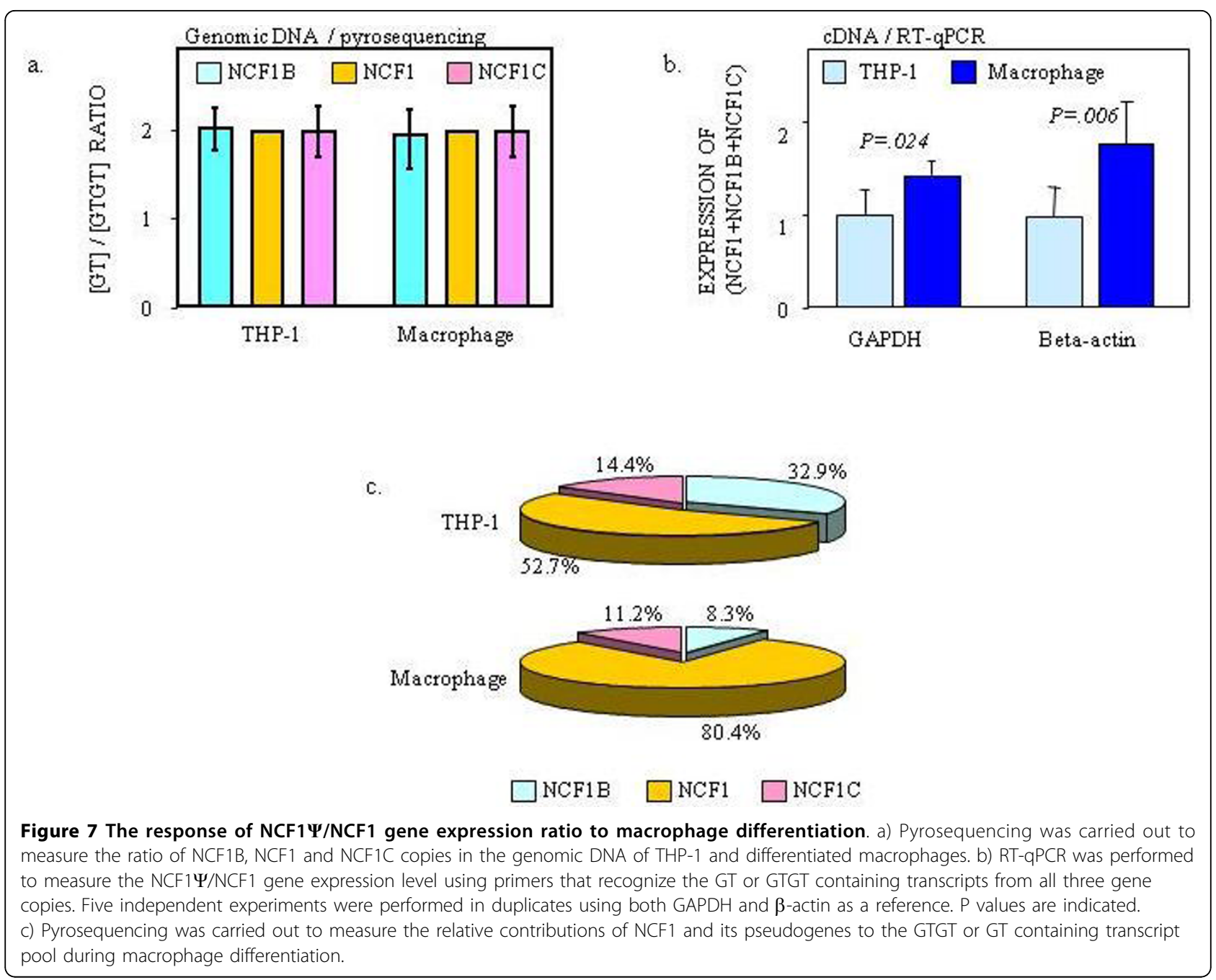

implies the existence of the NCF1 gene prior to the divergence of those two pseudogene duplicates, the NCF1 pseudogenes may emerge after the divergence of human and chimpanzee lineages. These data suggest that this NCF1 pseudogene CNV may be a consequence of recent gene duplications in human history [1].

Pseudogenes have long been considered to be 'dead' nonfunctional byproducts of genome evolution [29]. They were defined as genomic sequences that are similar to a functional gene but contain genetic defects that preclude the generation of functional products [30-32]. Recent findings have prompted to revise the definition of pseudogenes, which are now defined as genomic sequences that arise from functional genes but cannot encode the same type of functional products (i.e. protein, tRNA or rRNA) as the original genes [29]. Human genome is estimated to contain $\sim 20,000$ pseudogenes [33], it will be important to know how many and which pseudogenes are functional. Recently emphasis has been placed on polymorphisms such as CNV that has been documented to play a role in disease pathogenesis [34-39]. It has not been reported that a CNV of a pseudogene is biologically relevant. Historically, the NCF1 pseudogenes are considered "pseudo" because of their 2-bp GT deletion in exon 2, which is predicted to cause a frameshift and an early stop codon in protein synthesis. In our analysis of GT-containing transcript, the pseudogenes were far less active. However, our data revealed for the first time that a portion of NCF1 pseudogene transcripts do not utilize their defective exon-2, instead, they may use alternative exons to skip over their mutant exon-2 (Figure 6). When we measured transcript exclusive to exon-2, the NCF1 pseudogenes had varying transcriptional capacities that responded robustly to PMA induced macrophage differentiation (Figure 7) and showed distinct expression patterns in different human tissues (Figure 8). These observations prompted us to wonder if these NCF1 pseudogenes are "functional" or "unsuccessful duplicates". Recent studies have showed that $\sim 95 \%$ of multi-exon genes undergo tissue-specific 

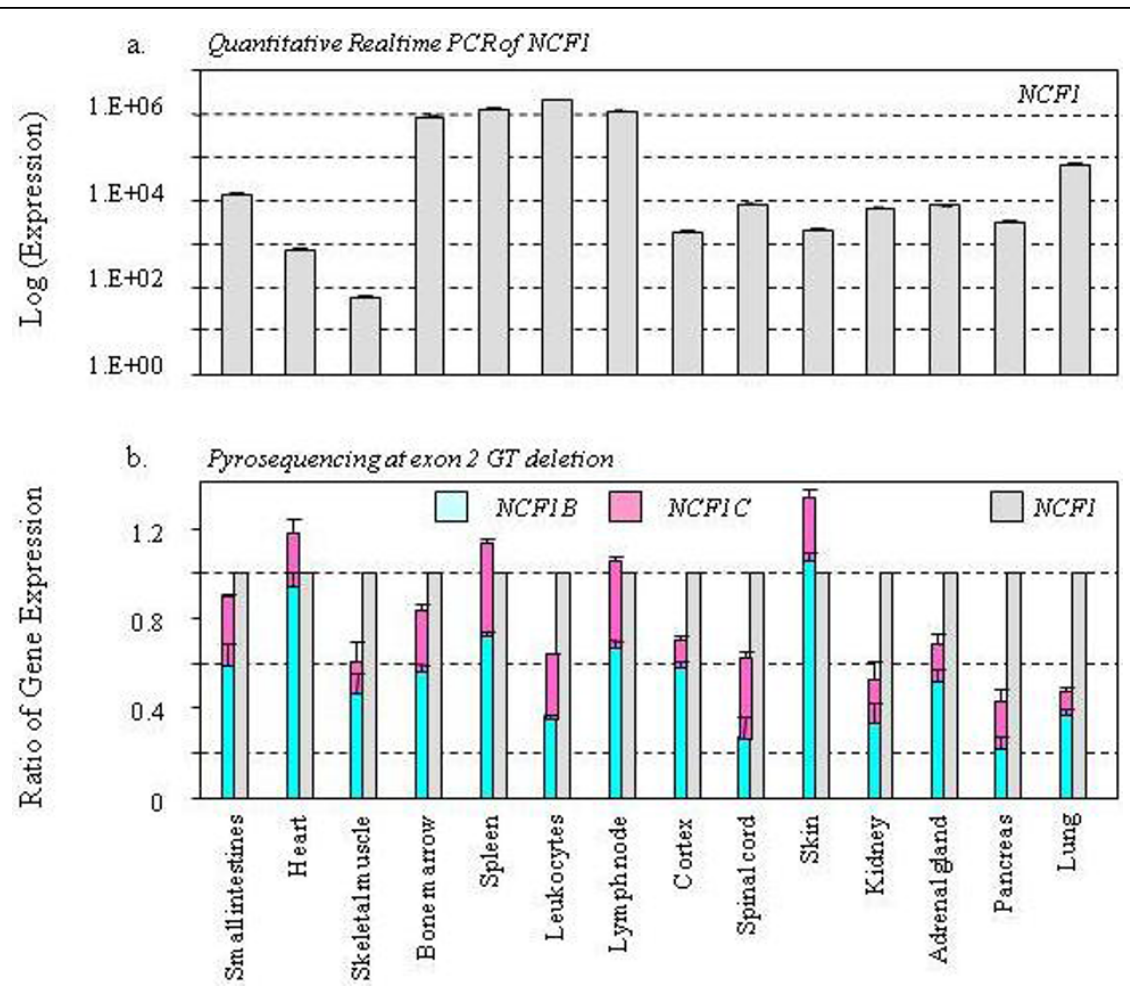

Figure 8 NCF1 1 /NCF1 gene expression in human tissues. a). RT-qPCR was performed using primers specific to the NCF1 gene to determine the NCF1 expression in different human tissues. The data is in logarithmic scale. b). Pyrosequencing was performed to determine the relative transcript levels of NCF1, NCF1B and NCF1C. Three independent experiments were performed in duplicates.

alternative splicing [40-42]; on the other hand, genes can also function by making regulatory non-coding RNAs in addition to making proteins [43]. These results certainly challenge the current perception of the NCF1 pseudogenes.

We observed differential expression of NCF1 and its pseudogenes and a varying contribution of the genes to the total transcript pool (Figure 5, 7, and 8). It may be caused by differential alternative splicing patterns, different promoter activities, and/or differential mRNA degradation. As indicated by sequence alignment with ClustalW2, the 5' untranslated regions of the NCF1 pseudogenes are nearly identical to the equivalent region of NCF1 (Additional file 7). Collectively, putative binding sites of 45 transcription factors (TFBS) were predicted with rVISTA (Additional file 7). The number and exact locations of these TFBS are also nearly identical among these three genes (Additional file 7), except that the NCF1 gene contains 19 AML1 TFBS and 4 CREB TFBS instead of the 18 AML1 sites and 3 CREB sites in both pseudogenes. Our data suggest that a portion of pseudogene transcripts did not adopt the GT-containing exon-2, which may explain our observation that NCF1 pseudogenes displayed fewer GT-containing transcripts relative to the true NCF1 gene. However, we are unclear if differential promoter activities and mRNA degradation contribute to this observation.

The CGD patients have shown that NCF1 is essential in the function of the neutrophil in the first line of host defense against many pathogenic bacteria and fungi. About $93 \%$ of humans patients caused by NCF1 mutations are homozygous for the 2-bp GT deletion [12,13]. Obviously the NCF1 pseudogenes, are not simply replacement duplicates, otherwise they may have compensated for the loss of NCF1 gene copies in the CGD patients. Two recent studies have reported that less copies of NCF1 pseudogenes may produce more reactive oxygen intermediates [44] and may exaggerate certain diseases involving inflammatory process such as inflammatory bowel disease [45]. Therefore, these two NCF1 pseudogenes may produce protein isoforms or small RNAs that act as inhibitors of the normal NCF1 function. Superoxide is a double-edged sword, it is essential for phagocytes to exert their bactericidal function, but in excess it is also toxic to our own cells. Cells in other tissues would benefit from the existence of these two pseudogenes if they could recognize different stimuli and then reduce the NCF1/NADPH oxidase activity and superoxide production accordingly. For example, they may help to reduce the inflammatory process during atherosclerosis. 


\section{Conclusions}

Taken together this study reported the existence of an NCF1 pseudogene CNV in three normal populations. These NCF1 pseudogenes are actively transcribed in human tissues. They can produce transcripts using alternative exon-2 instead of their defective exon-2. These results prompt us to re-consider their non-functional status in pseudogenes classification [29]. The functional contribution of the NCF1 pseudogenes to NCF1 function and the pathological relevance of this copy number variation will merit further investigations.

\section{Methods}

\section{Subjects and Materials}

Genomic DNAs of 86 unrelated individual DNA samples (The Human Variation Panels, 32 African-Americans [AA], 30 Caucasians [Cau], and 24 Mexicans [Mex]) and $2 \mathrm{CEPH/UTAH}$ pedigrees (1331 and 1362) were obtained from The Coriell Cell Repositories. Single-donor Epstein-Barr virus (EBV)-immortalized lymphoblastoid cell lines (LCLs) were obtained from two sources: The Coriell Cell Repositories and The Emory Zafari Collection (kindly provided by Dr. Zafari of Emory Cardiology) [46]. Genomic DNAs of 48 unrelated healthy individuals directly extracted from peripheral blood cells instead of LCLs were kindly provided by Dr. Kittner of University of Maryland [47]. A human monocyte cell line THP-1 was purchased from ATCC (TIB202). Total RNAs from human tissues were purchased from Clontech and US Biological. Phorbol 12-myristate 13-acetate (PMA) was purchased from Krackler Scientific (Cat. No. P1585). All subjects provided informed consent, and the study was approved by the Institutional Review Board of Morehouse School of Medicine.

\section{Cell Culture}

Lymphoblastoid cells and THP-1 cells were grown in RMPI-1640 supplemented with 10\% fetal bovine serum (FBS), $2 \mathrm{mM} \mathrm{L-glutamine,} \mathrm{and} \mathrm{1 \%} \mathrm{penicillin/streptomy-}$ cin. Cells were maintained in a humidified atmosphere containing $5 \% \mathrm{CO} 2$ at $37^{\circ} \mathrm{C}$. The differentiation of THP1 monocytes into macrophages was induced by incubation in $800 \mathrm{ng} / \mathrm{ml}$ of PMA for 12 hours. The non-adherent cells were removed by aspiration; adherent cells were allowed to differentiate for 3-4 days.

\section{Genomic DNA and Total RNA Extraction}

Genomic DNA was extracted from lymphoblastoid cells following a standard protocol described previously [48]. Total RNA was isolated using the RNeasy Mini kit (Qiagen). First strand cDNA was generated with $1 \mu \mathrm{g}$ of total RNA using the Superscript III-RT system (Invitrogen).

\section{Genotyping}

Genomic DNA and cDNA were subjected to genotyping at the 2-bp GT deletion in Exon-2 and a 1-bp difference in Exon-9 (Figure 1). PCR and pyrosequencing primers are provided in Additional file 8. These primers were designed to recognize both NCF1 and its pseudogenes without discrimination. The PCR products were examined by agarose gel electrophoresis to ensure PCR specificity. Genotyping was carried out with a pyrosequencer (PSQ96MA, Pyrosequencing, Uppsala, Sweden) following the manufacturer's protocol. Briefly, a $20 \mu \mathrm{L}$ PCR reaction was carried out with either genomic DNA or cDNA. PCR products were then denatured and singlestrand DNA templates were collected with streptavidincoated Dynabeads (Dynal, Oslo, Norway). A pyrosequencing primer was added and pyrosequencing was performed in an automated PSQ96MA instrument. The pyrogram peak heights were evaluated per individual to give an accurate count of allele ratios using the PyroMarkID Software v1.0 provided by the manufacturer.

\section{Quantitative Real-Time PCR (RT-qPCR)}

RT-qPCR was carried out using a LightCycler thermocycler (Roche) and a SYBR green kit (Roche). Oligo-dT primers were used in the reverse transcription, their sequences are provided in Additional file 8. Cycle numbers obtained at the log-linear phase of the reaction were plotted against a standard curve prepared with serially diluted control samples. Expressions of target genes were normalized by GAPDH and $\beta$-actin levels. The RT-qPCR amplification specificity of the NCF1-specific primer set is shown in Additional file 9.

\section{Statistical Analysis}

All data is expressed as Mean \pm Standard Deviation (SD). Student's $t$ test was used in the setting of multiple comparisons where the appropriate and statistical significance is defined as $p \leq 0.05$.

\section{Bioinformatic analysis}

Nucleotide sequences were retrieved from The UCSC Genome Browser (Human Assembly 2006 March). Sequence alignments were performed using multiple sequence alignment with MAP http://www.ebi.ac.uk/ Tools/clustalw2/index.html. Phylogenetic trees were constructed using MegAlign ClustalW of DNAStar Software

\footnotetext{
Additional file 1: Genotyped positions of cDNA amplicons used in copy number variation analysis. A) Amplicon generated at the exon-1 exon-2 boundary. B) Amplicon generated in exons 8 and 9. Genotyped locations are bold and underlined. Red base represents the end of an exon and blue represents the start of the neighboring exon. Click here for file

[http://www.biomedcentral.com/content/supplementary/1471-2156-1113-S1.JPEG ]
} 
Additional file 2: Representative pyrograms showing low pyrosequencing noise. a) The 2-bp GT deletion in exon 2. b) The A/G substitution in exon 9.

Click here for file

[http://www.biomedcentral.com/content/supplementary/1471-2156-1113-S2.JPEG ]

Additional file 3: CNV allele frequencies in three populations. Totally 86 non-related individuals (32 African-Americans, 30 Caucasians and 24 Mexicans) were genotyped at this CNV.

Click here for file

[http://www.biomedcentral.com/content/supplementary/1471-2156-1113-S3.XLS ]

Additional file 4: Copy number variation at the NCF1 locus observed in genomic DNA samples extracted directly from peripheral white blood cells. In order to eliminate the possibility that this CNV is an artefact caused by chromosomal instability in lymphoblastoid cell lines, we analyzed 48 genomic DNA samples directly extracted from human peripheral white blood cells. Means of 3 independent experiments performed in duplicate.

Click here for file

[http://www.biomedcentral.com/content/supplementary/1471-2156-1113-S4.JPEG]

Additional file 5: cDNA sequences of alternatively spliced exons. By $P C R$, cloning and direct DNA sequencing, we have experimentally discovered two novel alternative exons (GenBank: GU215077, GU215078) located in the intron-1. Neither of these two transcripts used the GTcontaining exon-2.

Click here for file

[http://www.biomedcentral.com/content/supplementary/1471-2156-1113-S5.JPEG ]

Additional file 6: Putative open reading frames of alternative spliced transcripts. The open reading frame (ORF) of two alternative spliced products, sub1 and sub2, were predicted with the NCBI ORF Finder.

Click here for file

[http://www.biomedcentral.com/content/supplementary/1471-2156-1113-S6.JPEG]

Additional file 7: Putative transcription factor binding sites (TFBS) of the human NCF1 gene and its pseudogenes. A $20 \mathrm{~kb}$ sequence of 5 '-flanking region of each of NCF1 and its pseudogenes (immediately upstream to exon 1) was retrieved from UCSC Genome Browser. Sequence alignment was performed with EMBL-EBI CLUSTAL 2.0.12 (Larkin et al., 2007). Putative TFBS was predicted with rVISTA (Loots et al., 2002) using the vertebrates TRANSFAC matrices and cut-off 1.0 for both matrix similarity and core similarity.

Click here for file

[http://www.biomedcentral.com/content/supplementary/1471-2156-1113-S7.DOC ]

Additional file 8: Primers used in this study. This table provides the detailed sequence information of oligonucleotides that were used in this study.

Click here for file

[http://www.biomedcentral.com/content/supplementary/1471-2156-1113-S8.XLS ]

Additional file 9: Melting curves of quantitative real-time PCR (RT$q P C R)$ using primers specific for the true $p 47^{\text {phox }}$ mRNA. The specificity of this primer set is indicated the single sharp peak. The negative control is indicated by the straight line.

Click here for file

[http://www.biomedcentral.com/content/supplementary/1471-2156-1113-S9.JPEG ]

\section{Acknowledgements}

The authors would like to take the opportunity to thank Gary H. Gibbons, Maziar Zafari, Sandra Harris-Hooker, Mukaila Akinbami, David B. Allison, and Kathy K. Griendling Taylor for their scientific comments. This work was supported by grants of American Heart Association (09GRNT2300003) and NIH (NIH/NHLBI T32HL067702, HL003676, HL095098, NIH/NCRR RR014758 and RR003034, NIH/NIGMS HL095098). The research was conducted in a facility constructed with support from a Research Facilities Improvement Grant (NIH/NCRR RR07571). This work is also supported in part by the Baltimore Research Enhancement Award Program in Stroke and the Baltimore Geriatrics Research, Education, and Clinical Center of the Department of Veterans Affairs.

\section{Authors' contributions}

TB carried out all experiments, participated in study design and drafted the manuscript. QW assisted experiments. IC contributed to the monocyte experiments. QS designed the study, carried out the computational analysis in sequence alignment, ORF prediction and TFBS prediction. QS revised the manuscript. All authors have read and approved the final version of the manuscript.

Received: 7 August 2009 Accepted: 23 February 2010 Published: 23 February 2010

\section{References}

1. Korbel JO, Kim PM, Chen X, Urban AE, Weissman S, Snyder M, Gerstein MB: The current excitement about copy-number variation: how it relates to gene duplications and protein families. Curr Opin Struct Biol 2008, 18(3):366-374

2. Cooper GM, Nickerson DA, Eichler EE: Mutational and selective effects on copy-number variants in the human genome. Nat Genet 2007, 39(7 Suppl):S22-29.

3. Feuk $L$, Carson AR, Scherer SW: Structural variation in the human genome. Nat Rev Genet 2006, 7(2):85-97.

4. Korbel JO, Urban AE, Affourtit JP, Godwin B, Grubert F, Simons JF, Kim PM, Palejev D, Carriero NJ, Du L, et al: Paired-end mapping reveals extensive structural variation in the human genome. Science 2007, 318(5849):420-426

5. Nguyen DQ, Webber C, Ponting CP: Bias of selection on human copynumber variants. PLoS Genet 2006, 2(2):e20

6. Redon $\mathrm{R}$, Ishikawa S, Fitch KR, Feuk L, Perry GH, Andrews TD, Fiegler $H$, Shapero MH, Carson AR, Chen W, et al: Global variation in copy number in the human genome. Nature 2006, 444(7118):444-454

7. Sebat J, Lakshmi B, Troge J, Alexander J, Young J, Lundin P, Maner S, Massa H, Walker M, Chi M, et al: Large-scale copy number polymorphism in the human genome. Science 2004, 305(5683):525-528.

8. Tuzun E, Sharp AJ, Bailey JA, Kaul R, Morrison VA, Pertz LM, Haugen E, Hayden $\mathrm{H}$, Albertson D, Pinkel $\mathrm{D}$, et al: Fine-scale structural variation of the human genome. Nat Genet 2005, 37(7):727-732.

9. Volpp BD, Nauseef WM, Donelson JE, Moser DR, Clark RA: Cloning of the CDNA and functional expression of the 47-kilodalton cytosolic component of human neutrophil respiratory burst oxidase. Proc Natl Acad Sci USA 1989, 86(18):7195-7199.

10. El-Benna J, Dang PM, Gougerot-Pocidalo MA, Marie JC, Braut-Boucher F: p47phox, the phagocyte NADPH oxidase/NOX2 organizer: structure, phosphorylation and implication in diseases. Exp Mol Med 2009, 41(4):217-225.

11. Rada B, Leto $T L$ : Oxidative innate immune defenses by Nox/Duox family NADPH oxidases. Contrib Microbiol 2008, 15:164-187.

12. Casimir CM, Bu-Ghanim HN, Rodaway AR, Bentley DL, Rowe P, Segal AW: Autosomal recessive chronic granulomatous disease caused by deletion at a dinucleotide repeat. Proc Natl Acad Sci USA 1991, 88(7):2753-2757.

13. Noack D, Rae J, Cross AR, Ellis BA, Newburger PE, Curnutte JT, Heyworth PG: Autosomal recessive chronic granulomatous disease caused by defects in NCF-1, the gene encoding the phagocyte p47-phox: mutations not arising in the NCF-1 pseudogenes. Blood 2001, 97(1):305-311.

14. Barry-Lane PA, Patterson C, Merwe van der M, Hu Z, Holland SM, Yeh ET, Runge MS: p47phox is required for atherosclerotic lesion progression in ApoE(-/-) mice. J Clin Invest 2001, 108(10):1513-1522.

15. Griendling KK, Sorescu D, Ushio-Fukai M: NAD(P)H oxidase: role in cardiovascular biology and disease. Circ Res 2000, 86(5):494-501.

16. Hsich E, Segal BH, Pagano PJ, Rey FE, Paigen B, Deleonardis J, Hoyt RF, Holland SM, Finkel T: Vascular effects following homozygous disruption of p47(phox): An essential component of NADPH oxidase. Circulation 2000, 101(11):1234-1236. 
17. Kirk EA, Dinauer MC, Rosen H, Chait A, Heinecke JW, LeBoeuf RC: Impaired superoxide production due to a deficiency in phagocyte NADPH oxidase fails to inhibit atherosclerosis in mice. Arterioscler Thromb Vasc Biol 2000, 20(6):1529-1535

18. Landmesser U, Cai H, Dikalov S, McCann L, Hwang J, Jo H, Holland SM, Harrison DG: Role of p47(phox) in vascular oxidative stress and hypertension caused by angiotensin II. Hypertension 2002, 40(4):511-515.

19. Thomas M, Gavrila D, McCormick ML, Miller FJ Jr, Daugherty A, Cassis LA, Dellsperger KC, Weintraub NL: Deletion of p47phox attenuates angiotensin II-induced abdominal aortic aneurysm formation in apolipoprotein E-deficient mice. Circulation 2006, 114(5):404-413.

20. Francke U, Hsieh CL, Foellmer BE, Lomax KJ, Malech HL, Leto TL: Genes for two autosomal recessive forms of chronic granulomatous disease assigned to 1q25 (NCF2) and 7q11.23 (NCF1). Am J Hum Genet 1990, 47(3):483-492.

21. Baumer A, Dutly F, Balmer D, Riegel M, Tukel T, Krajewska-Walasek M, Schinzel AA: High level of unequal meiotic crossovers at the origin of the 22q11. 2 and 7q11.23 deletions. Hum Mol Genet 1998, 7(5):887-894.

22. Del Campo M, Antonell A, Magano LF, Munoz FJ, Flores R, Bayes M, Perez Jurado LA: Hemizygosity at the NCF1 gene in patients with WilliamsBeuren syndrome decreases their risk of hypertension. Am J Hum Genet 2006, 78(4):533-542.

23. Gorlach A, Lee PL, Roesler J, Hopkins PJ, Christensen B, Green ED, Chanock SJ, Curnutte JT: A p47-phox pseudogene carries the most common mutation causing p47-phox- deficient chronic granulomatous disease. J Clin Invest 1997, 100(8):1907-1918.

24. Roesler J, Curnutte JT, Rae J, Barrett D, Patino P, Chanock SJ, Goerlach A: Recombination events between the p47-phox gene and its highly homologous pseudogenes are the main cause of autosomal recessive chronic granulomatous disease. Blood 2000, 95(6):2150-2156.

25. Heyworth PG, Noack D, Cross AR: Identification of a novel NCF-1 (p47-phox) pseudogene not containing the signature GT deletion: significance for A47 degrees chronic granulomatous disease carrier detection. Blood 2002, 100(5):1845-1851.

26. Gruber JD, Colligan PB, Wolford JK: Estimation of single nucleotide polymorphism allele frequency in DNA pools by using Pyrosequencing. Hum Genet 2002, 110(5):395-401.

27. Jeon JP, Shim SM, Nam HY, Baik SY, Kim JW, Han BG: Copy number increase of $1 \mathrm{p} 36.33$ and mitochondrial genome amplification in EpsteinBarr virus-transformed lymphoblastoid cell lines. Cancer Genet Cytogenet 2007, 173(2):122-130.

28. Boyle JJ: Macrophage activation in atherosclerosis: pathogenesis and pharmacology of plaque rupture. Curr Vasc Pharmacol 2005, 3(1):63-68.

29. Zheng D, Gerstein MB: The ambiguous boundary between genes and pseudogenes: the dead rise up, or do they? Trends Genet 2007, 23(5):219-224.

30. Balakirev ES, Ayala FJ: Pseudogenes: are they "junk" or functional DNA? Annu Rev Genet 2003, 37:123-151.

31. Mighell AJ, Smith NR, Robinson PA, Markham AF: Vertebrate pseudogenes. FEBS Lett 2000, 468(2-3):109-114.

32. Vanin EF: Processed pseudogenes: characteristics and evolution. Annu Rev Genet 1985, 19:253-272.

33. Torrents D, Suyama M, Zdobnov E, Bork P: A genome-wide survey of human pseudogenes. Genome Res 2003, 13(12):2559-2567.

34. Henrichsen $C N$, Chaignat $E$, Reymond A: Copy number variants, diseases and gene expression. Hum Mol Genet 2009, 18(R1):R1-8.

35. Ionita-Laza I, Rogers AJ, Lange C, Raby BA, Lee C: Genetic association analysis of copy-number variation (CNV) in human disease pathogenesis. Genomics 2009, 93(1):22-26.

36. Lanktree $M$, Hegele RA: Copy number variation in metabolic phenotypes. Cytogenet Genome Res 2008, 123(1-4):169-175.

37. McCarroll SA: Extending genome-wide association studies to copynumber variation. Hum Mol Genet 2008, 17(R2):R135-142.

38. Ptacek T, Li X, Kelley JM, Edberg JC: Copy number variants in genetic susceptibility and severity of systemic lupus erythematosus. Cytogenet Genome Res 2008, 123(1-4):142-147.

39. Schaschl H, Aitman TJ, Vyse TJ: Copy number variation in the human genome and its implication in autoimmunity. Clin Exp Immunol 2009, 156(1):12-16.
40. Pan Q, Shai O, Lee LJ, Frey BJ, Blencowe BJ: Deep surveying of alternative splicing complexity in the human transcriptome by high-throughput sequencing. Nat Genet 2008, 40(12):1413-1415.

41. Castle JC, Zhang C, Shah JK, Kulkarni AV, Kalsotra A, Cooper TA, Johnson JM: Expression of 24,426 human alternative splicing events and predicted cis regulation in 48 tissues and cell lines. Nat Genet 2008, 40(12):1416-1425.

42. Wang ET, Sandberg R, Luo S, Khrebtukova I, Zhang L, Mayr C, Kingsmore SF, Schroth GP, Burge CB: Alternative isoform regulation in human tissue transcriptomes. Nature 2008, 456(7221):470-476.

43. Eddy SR: Non-coding RNA genes and the modern RNA world. Nat Rev Genet 2001, 2(12):919-929.

44. Greve B, Hoffmann P, Vonthein R, Kun J, Lell B, Mycko MP, Selmaj KW, Berger K, Weissert R, Kremsner PG: NCF1 gene and pseudogene pattern: association with parasitic infection and autoimmunity. Malar J 2008, 7:251.

45. Harbord M, Hankin A, Bloom S, Mitchison H: Association between p47phox pseudogenes and inflammatory bowel disease. Blood 2003, 101(8):3337.

46. Zafari AM, Davidoff MN, Austin H, Valppu L, Cotsonis G, Lassegue B, Griendling KK: The A640G and C242T p22(phox) polymorphisms in patients with coronary artery disease. Antioxid Redox Signal 2002, 4(4):675-680

47. Song Q, Cole JW, O'Connell JR, Stine OC, Gallagher M, Giles WH, Mitchell BD, Wozniak MA, Stern BJ, Sorkin JD, et al: Phosphodiesterase 4D polymorphisms and the risk of cerebral infarction in a biracial population: the Stroke Prevention in Young Women Study. Hum Mol Genet 2006, 15(16):2468-2478

48. Song Q, Chao J, Chao L: DNA polymorphisms in the 5 '-flanking region of the human tissue kallikrein gene. Hum Genet 1997, 99(6):727-734.

doi:10.1186/1471-2156-11-13

Cite this article as: Brunson et al:: A copy number variation in human NCF1 and its pseudogenes. BMC Genetics 2010 11:13.

\section{Submit your next manuscript to BioMed Central and take full advantage of:}

- Convenient online submission

- Thorough peer review

- No space constraints or color figure charges

- Immediate publication on acceptance

- Inclusion in PubMed, CAS, Scopus and Google Scholar

- Research which is freely available for redistribution

Submit your manuscript at www.biomedcentral.com/submit
C Biomed Central 